\title{
INTERFEROMETRIC MEASUREMENTS OF BINARIES
}

\author{
H.A. MCALISTER
}

CHARA, Georgia State University, Atlanta, Georgia, USA

\section{Introduction}

This paper is a brief overview of the past performance and future promise of interferometry as applied to the study of binary stars. For the sake of brevity, the important results from infrared techniques in recent years are regretfully omitted here. It is probably unnecessary to remind the reader that the analysis of binary star orbital motions is the only direct means for the determination of stellar masses. Historically, stellar masses have resulted primarily from orbits that are sufficiently wide in angular separation to permit the astrometric determination of the motions of the individual components about the center of mass as well as the parallax of the system or from short-period spectroscopic/eclipsing binaries. The advent of long baseline optical interferometers holds the promise of a wealth of new stellar mass data through the direct resolution of spectroscopic binaries.

Interferometry in the context of binaries is a very narrow-field, differential astrometric method. It is time for formal reconsideration of such terms as "visual" and "astrometric" binaries, but we must resist the temptation to compound the problem by introducing the term "interferometric" binary.

\section{Techniques and Milestones}

The application of Michelson's then newly proposed technique of astronomical interferometry to the study of double stars was first proposed more than a century ago by Karl Schwarzschild (1895). He described an adjustable objective grating method for obtaining and varying the spacing of interferometric fringes whose variable visibility would tell the observer the orientation and separation of the components of a binary. The first important application of Michelson interferometry in the field was made by Anderson (1920) and Merrill (1922) who used an adjustable slit device on 
the 100-inch Hooker telescope on Mt. Wilson to measure Capella and other close binaries and inspected a number of spectroscopic binaries for possible resolution. Their measures are noteworthy for extreme accuracy, and the combination of the Mt. Wilson measures of Capella with subsequent speckle data obtained many revolutions later was instrumental in providing an orbit of extreme accuracy for that classic "friend of the interferometrist."

By far, the most significant interferometric contribution prior to the discovery of speckle interferometry was the work of William S. Finsen at the Johannesburg Observatory. Finsen designed and fabricated with his own hands an "eyepiece interferometer." For more than two decades, Finsen proceeded to discover numerous new, close binaries and to measure then with high accuracy. At the end of his career, he gave a sober evaluation of the method describing its virtues but warning against its susceptibility to misuse by incautious observers (Finsen 1971). The work of Finsen in South Africa and Anderson and Merrill on Mt. Wilson provide fine examples of binary star measures whose time dependent quality cannot be entirely superseded by observations at subsequent epochs.

Even if this meeting were not being held in honor of Hanbury Brown, it would be appropriate to emphasize the significance of the observations of the Spica system in 1966 and 1970 from the stellar intensity interferometer at Narrabri, New South Wales (Herbison-Evans et al. 1971). Their elegant combination of interferometric, spectroscopic and photometric data not only yielded a rare determination of the masses of early spectral type stars, but also determined the parallax and luminosities of the components as well as the linear radius of the primary. This landmark achievement will not be duplicated until the present generation of long-baseline interferometers become fully operational during the next few years.

Labeyrie's invention of speckle interferometry in 1970 provided a wonderful new tool for the measurement of binary stars with angular separations limited by the Airy criteria of whatever large telescopes could be brought to bear. A number of speckle programs which emphasized double stars were originated in France, Britain, Germany, Russia, and the U.S., with the majority of the results generated by the author's own program, which is now under the direction of William Hartkopf, at Georgia State University. Rather than give what would be a very incomplete bibliography, the reader is referred to the regularly updated Catalogue of Interferometric Measurements of Binary Stars accessible at http://www.chara.gsu.edu. While great efforts were expended over the years to make speckle interferometry a widely applicable method for high resolution astronomical imaging, the technique is ideally suited to binary star measurement. The CHARA program is now the longest, continuously pursued program of interferometric studies of binaries. In 1990, the U.S. Naval Observatory ini- 
tiated a binary star speckle interferometry program using the 26-inch refractor in Washington, and the first results of this highly productive effort should appear in the literature in 1997.

Speckle interferometry is presently being surpassed in its ability to resolve close binaries by long-baseline interferometers. These instruments employ the technique of amplitude interferometry over baselines of tens to hundreds of meters to achieve resolutions ranging from a few milliarcseconds to perhaps 100 microarcseconds. The greatest body of binary star data to date using such interferometers comes from the Mark III interferometer built jointly by the Naval Research Laboratory and the USNO and operated on Mt. Wilson from the mid-1980's until the early 1990's. The results from the Mark III have been summarized at this meeting in the poster presentation by Christian Hummel. The Mark III provided considerable overlap with speckle interferometry, and the comparison of the two data types on the same system shows the clear advantage in obtaining higher accuracy by going to longer baselines. This is particularly demonstrated in the Mark III orbit of Capella (Hummel et al. 1994)

\section{Advantages and Performance Limits}

In comparison with classical visual methods for studying binary stars, interferometry offers the dual advantages of increased resolution and increased accuracy. While skilled visual micrometrists (currently comprising a singledigit sub-population of astronomers) can measure binaries with separations perhaps as small as 0.15 to 0.12 arcseconds, these measures tend to be qualitative rather than quantitative. Using 4-m class telescopes, speckle observers can achieve limiting resolutions of 0.030 arcsecond with an accuracy of perhaps $10 \%$ in a single measurement. Visual observers have occasionally complained of unfair comparison, pointing out that they are using aperture five or six times smaller than the typical telescope used by speckle teams. However, the much larger number of speckles encountered at these large apertures leads this writer to believe that micrometer measures at 4-m telescopes would be very difficult to undertake. Regrettably, this assertion has never been tested. For binaries which are well resolved by both techniques, speckle clearly has the advantage in accuracy as can be immediately seen by perusal of the orbits calculated by Hartkopf et al. (1989).

Interferometric measurements also have the potential for providing accurate measurements of the intensity ratios of the components. In terms of placing points on the empirical mass-luminosity relation, $\Delta \mathrm{m}$ is as important as the astrometric orbit. It has proven challenging to extract this photometric information reliably from speckle data, however. It is most 
certainly not feasible to measure intensity ratios from Labeyrie's classical approach to analyzing speckle data, and although $\Delta \mathrm{m}$ 's have been published which have resulted from image reconstruction algorithms, aperture masking or triple correlation analysis, their reliability has not been verified through external comparisons. It therefore remains a challenge to single aperture interferometrists to prove and exploit the potential in this important area. The extraction of intensity ratios from amplitude interferometry is dependent upon the accurate calibration of visibilities, but may ultimately be more straightforward than the efforts exerted on speckle data.

The $\Delta$ m detection limits of various techniques are also an important point for comparison. An approximate but reasonable expectation from speckle methods is 3-4 magnitudes while one might hope that long-baseline interferometers can perhaps go one magnitude greater in limiting $\Delta \mathrm{m}$. These limits are dependent on the combined magnitude of the target and rapidly approach zero as the object brightness approaches the limiting magnitude of the technique. Speckle interferometry with photon counting detectors is perhaps limited to objects brighter than $\mathrm{V}=+12$ while interferometric arrays are not likely to reach such a limit without very sophisticated adaptive optics and moderately large apertures.

\section{Resolution of Spectroscopic Binaries}

The resolution of spectroscopic binaries by interferometric techniques provides a powerful combination of complementary capabilities. Traditionally, few spectroscopic binaries have been directly resolved because classical astrometric techniques do not possess the resolution required for objects in the period domain to which spectroscopy is normally sensitive. When the happy circumstance of having a resolved spectroscopic binary does arrive, the interferometrically determined orbital semi-major axis and inclination combine with the spectroscopically determined $a \sin i$ and mass ratio to yield masses, distance and luminosities. The distance thus determined is referred to as the "orbital parallax."

A conservative estimate of the productivity of interferometer arrays in this regard is seen by noting that 180 double-lined systems from the "Eighth Catalogue of the Orbital Elements of Spectroscopic Binary Systems" (Batten et al. 1989) have predicted angular separations exceeding 1 milliarcsec. Recognizing that several interferometers will have limiting resolutions 10 times small than this value, literally hundreds of double-lined pairs will be resolved. This will completely close the gap between the "visual" and "spectroscopic" binaries and provide a wealth of new data for the mass/luminosity and mass/radius relations.

For single-lined systems, two directions of progress are noteworthy. First, 
techniques like tomographic separation of spectra (Bagnuolo et al. 1994) and the two-dimensional, cross-correlation method of Zucker and Mazeh (1994) are turning single-lined systems into double-lined pairs with known mass ratios. The latter technique is currently being applied in a series of papers in which Hyades spectroscopic binaries resolved by lunar occultation observations, speckle interferometry and the Mark III interferometer are finally giving up their masses and new geometrically determined distances to the cluster. Secondly, at the time of this writing, we anticipate the release of new parallax data from the HIPPARCOS satellite. If these parallaxes are of sufficient accuracy and free from the effects of orbital submotions, they will provide the missing information needed to make resolved single-lined binaries give up their secrets.

\section{The Third CHARA Catalogue}

The continuously updated "Catalogue of Interferometric Measurements of Binary Stars," maintained on-line at CHARA by William Hartkopf, is perhaps the best summary of the current status of the field. This catalogue includes speckle data as well as data from the modern generation of optical arrays which at present is limited to the Mark III. Also included are occultation measurements (because of their strong overlap with interferometry) and measurements from other amplitude interferometers except for the large body of work from Finsen's eyepiece interferometer. Relevant statistics from the catalogue are shown in Table 1 below. The column head "current" indicates the present status while the subsequent two columns include new data anticipated from the USNO and CHARA programs during 1997. The final column indicates the anticipated status of the catalogue after those results are included. Hopefully, additional material will be provided by other practitioners of interferometry.

\section{Conclusion}

During the past 20 years, speckle interferometry has essentially replaced visual micrometry as the preferred technique for measuring the angular motions in resolved binary systems. This transition is perhaps best represented by Charles Worley's decision in the late 1980's to set aside his micrometer and develop a speckle program at the USNO on the same telescope where he had for years applied his "double star eye."

Binary star observations will be a major part of the observing program at Georgia State University's CHARA Array now under construction on Mt. Wilson, California. This facility will employ five 1-meter aperture telescopes in a Y-shape configuration to achieve limiting resolutions of 200 microarcseconds at the $\mathrm{V}$ spectral bandpass and 1 milliarcsecond at the $\mathrm{K}$ 
TABLE 1. CHARA's Catalogue of Interferometric Measurements of Binary Stars

\begin{tabular}{lcccc}
\hline \multicolumn{1}{c}{ Category } & Current & USNO-97 & CHARA-97 & New \\
\hline No. of Resolved Stars & 3,455 & 467 & 1,146 & 4,000 \\
No. of Unresolved Stars & 2,843 & 0 & 197 & 3,012 \\
No. of New Resolved Binaries & 375 & 0 & 5 & 380 \\
No. of Measurements & 17,500 & 2,329 & 2,981 & 22,810 \\
CHARA & 13,408 & - & 2,981 & 16,389 \\
Occultation & 695 & - & - & 629 \\
Other & 3,397 & 2,329 & - & 5,726 \\
Median Separation (arcsec) & 0.25 & 1.14 & 0.45 & 0.31 \\
No. of Negative Results & 6,610 & 0 & 316 & 6,926 \\
CHARA & 4,816 & - & 316 & 5,132 \\
Other & 1,794 & 0 & - & 1,794 \\
Contributing Papers & 201 & 1 & 3 & 205 \\
\hline
\end{tabular}

band. It can be stated with confidence that binaries will also be emphasized at the Navy's Prototype Optical Interferometer (NPOI) on Anderson Mesa, Arizona, as well as by our host's facility near Narrabri, the Sydney University Stellar Interferometer (SUSI). These powerful new instruments will yield masses, distances, luminosities, effective temperatures and physical radii in unprecedented numbers for stars of almost all MK spectral types. The interferometric revolution in the field of binary star astrometry will be complete by the end of the century.

\section{References}

\section{References}

Anderson, J.A. (1920) Astrophys. Journ., 51, p. 263.

Bagnuolo, W.G., Gies, D.R., Hahula, M.J. and R. Wiemker, R. (1994) Astrophys. Journ., 423, p. 446.

Finsen, W.S. (1971) Astrophysics and Space Science, 11, p. 13.

Hartkopf, W.I., McAlister, H.A. and Franz, O.G. (1989) Astron. Journ., 98, p. 1014.

Herbison-Evans, D., Hanbury Brown, R., Davis, J., and Allen, L.R. (1971) Mon. Not. Roy. Astron. Soc., 151, p. 161.

Hummell, C.A., Armstrong, J.T., Quirrenbach, A., Buscher, D.F., Mozurkewich, D. and Elias, N.M. (1994) Astron. Journ., 107, p. 1859.

Merrill, P.W. (1922) Astrophys. Journ., 56, p. 43.

Schwarzschild, K. (1895) Astronomische Nachrichten, 139, p. 23.

Zucker, S. and Mazeh. T. (1994) Astrophys. Journ., 420, p. 806. 